\title{
The Effect of Pencil Beam, Collapsed Cone, and Monte Carlo Algorithms on Dose-Volume Parameters in Esophagus Cancer: The Digital Phantom Study
}

\author{
(D) Taylan BÜKÜLMEZ, ${ }^{1}$ ํ) Beyza Şirin ÖZDEMIR² \\ 'Department of Radiation Oncology, Van Regional Training and Research Hospital, Van-Turkey \\ ${ }^{2}$ Department of Radiation Oncology, Akdeniz University Faculty of Medicine, Antalya-Turkey
}

\begin{abstract}
OBJECTIVE
The aim of the study was to compare the effects of three different planning algorithms in esophageal cancer on treatment and normal tissues according to different energy and field angles.

\section{METHODS}

In this study, a tumor volume was determined in the middle esophagus in the digital phantom. By targeting this tumor volume, a three-dimensional conformal radiotherapy treatment plan was created and dose-volume histograms (DVH) were compared according to the Pencil Beam, Collapsed Cone,and Monte Carlo algorithms. The total dose was determined as 5040cGy (1.8 Gy/fraction). In DVH; mean planning target volume dose was evaluated as D50, D98, D2; mean dose for the heart as V5, V30; mean dose for lung as V5, V20; and also the maximum dose (Dmax) for the spinal cord and homogeneity index were assessed. A total of 18 plans created at the same energy levels ( 6 and $18 \mathrm{MV})$ and angles $(3,4$, and 5 fields) were compared using these three different algorithms.
\end{abstract}

\section{RESULTS}

Different algorithms created significant differences with the same energy and same field angles as we expected. Especially when considered in terms of normal tissues, the remarkable difference was in the heart (Dmean, V5), lung (Dmean, V5, V20), and spinal cord Dmax values. There were also differences in algorithms between PTV dose values. We found that with the increase in the energy level and field, the dose differences between algorithms significantly reduced.

\section{CONCLUSION}

Variations between algorithms that may occur due to the difference in density between tissues in the thoracic region should be taken into consideration.

Keywords: Collapsed cone; digital phantom; esophageal cancer; monte carlo; pencil beam.

Copyright $\odot$ 2021, Turkish Society for Radiation Oncology

\section{Introduction}

The purpose of radiotherapy (RT) is to protect the normal tissues organs at risk (OARs) as much as possible while providing an adequate treatment dose in the target tissue. In that case, the accuracy of the absorbed dose delivery is important for both normal tissues and target volume. As we know, different tissues and different organs in the human body have different physical and radiological features. This results in differential transport and absorption of photons and electrons in each tissue or organ. In clinical applications, inhomogeneous 
environments caused by air cavity, lung, bone, and high-density environments cause difficulties in calculating the dose distribution. Especially, developments in the calculated dose have been indicated when the extension of the irradiated volume is limited and low densities are present in or adjacent to the fields.[1-3] In addition, it is emphasized that in advanced RT techniques such as Intensity-modulated radiotherapy (IMRT), simple algorithms should be avoided for accurate dosimetry of small areas within and near inhomogeneities.[4] That means dose calculation algorithms used in treatment planning systems (TPSs) have advantages and disadvantages in calculating the absorbed dose in different regions. An ideal dose calculation algorithm can perfectly reflect the actual dose distribution and so reduces the uncertainty during the evaluation of treatment plans. According to the International Radiation Units Commission (ICRU Report 24) criteria, the dose calculation accuracy is required to be within 5\% (2-3\% may be advantageous in some situations).[5] In addition, the accuracy of a dose calculation algorithm has been proposed to be $2 \%$ on the low dose gradient or $2 \mathrm{~mm}$ on the high dose gradient.[6]

With the occurrence of different algorithms in TPSs in the past decade in some studies, the radiobiological and dosimetric impact of Pencil Beam (PB) versus Monte Carlo (MC) dose algorithms were evaluated. $[7,8]$ They reported MC algorithm was superior in terms of local control.

In our study, the esophagus was preferred due to its location in a heterogeneous region and $\mathrm{PB}$, Collapsed Cone (CC), and MC algorithms are compared dosimetrically. The aim is to show the differences between algorithms in a heterogeneous environment, and consequentially to emphasize that it may have a significant impact on future treatment plans.

\section{Materials and Methods}

This research is a digital phantom study. Patient data were not used in this study (It was done virtually over a computer system). As target, planning target volume (PTV), we identified was taken into account. PTV was determined for the tumor that was created virtually in the middle esophagus localization in the mathematical digital phantom.

The reason for choosing the middle esophagus as the primary tumor site is that this area is highly heterogeneous due to the presence of lung, heart, costa, vertebra, and spinal cord. In addition to PTV, critical organs such as lungs, spinal cord, and heart were contoured. The plans were created using the same target volume and critical structure contours in all plans for an effective comparison. In particular, we would like to point out that proving the accuracy of these three algorithms is not among our aims. The planning was prepared by protecting the dose constant as if the daily fraction dose was delivered to the real patient with 28 fractions of $180 \mathrm{cGy}$. Segments and wedge were not used in the plans. It was not tried to choose ideal angles to protect the organs. We, the physicians, determined the angles $(120,90$, and 72$)$, fields ( 3,4 , and 5$)$, energies (6-18MV), and then a total of 18 plans were investigated, according to $\mathrm{PB}, \mathrm{CC}$, and $\mathrm{MC}$ algorithms.

Ninety-five percent of PTV was covered by prescription isodose. On the (DVH), we evaluated D50, D98, D2, and mean doses for the PTV; mean dose, V5, V30 for the heart; mean dose, V5, V20 for lung; and Dmax for the spinal cord. In addition, we also compared the homogeneity index (HI) which evaluates the homogeneity of the dose distribution of the PTV. HI was calculated according to ICRU Report62 ([D2D98]/D50). Dose differences between PB, CC, and MC algorithms were examined.

\section{Monaco TPS}

Monaco TPS developed by Elekta Company was used in this study. There are manual and automatic contouring tools in the contouring part where the target volumes and OARs are drawn and their boundaries are determined. In the planning section, there are tools by which parameters such as the beam's gantry angle and Multi-Leaf Collimator can be manipulated. By changing and editing these parameters, OARs are preserved while giving the desired dose to the target volume. Monaco has the capability to calculate modalities such as photons, electrons, and protons beams.

For these calculations, the $\mathrm{PB}, \mathrm{CC}$, and MC algorithms can be used by the user.

When the user starts to plan, the user chooses the algorithm to be calculated after selecting the number of beams, gantry angles of the beams, beam energy, etc.In three-dimensional conformal radiotherapy (3D-CRT) plans, the user defines a prescribed dose for the target region then the calculation starts. After the calculation is completed some hot and cold dose regions could appear in the treated volume. The user needs to adjust them manually. In IMRT and volumetric modulated arc therapy (VMAT) plans, this is done by Monaco according to the optimization parameters created by the user. The user adds the drawn structures to the optimization at this stage and defines the cost functions is available in the system to the optimization according to the type 
of these structures (Target, OAR). By manipulating the dose distribution with these parameters, while taking the maximum dose of the target volumes, it is ensured that the OARs are affected to the minimum from this dose. Monaco TPS has two optimization modes named "Pareto" and "Constrained." In the Pareto mode, the cost functions that aim to deliver the prescribed dose to the target volume are more powerful than the cost functions that try to keep the dose received by OARs within a certain limit. In other words, priority is given to target volumes in this mode. In the other mode, constrained, the situation is the opposite, that is, priority belongs to OARs. The constrained mode is used in all plans in this study.

\section{Mathematical Digital Phantom}

Various mathematical and statistical methods such as $\mathrm{MC}$ are used to calculate the behavior of radiation in the human body. Various software uses these methods and it has been ensured that radiation transport calculations such as dose and flux distribution are performed in computer environment. This software simulates the interaction of radiation with matter using cross section data. Cross-section data include the probability distribution of various events that are likely to occur as a result of the interaction by defining each isotope depending on the type and energy of the radiation. For this reason, to simulate the interaction of radiation with matter, the environment must be described in detail at the atomic scale.

Various mathematical models and phantoms have been produced to perform radiation transport calculations in computer environment. In the past, these models have been defined using simple geometric shapes such as square, rectangular, ellipse, and cylinder medical internal radiation dose (Fig. 1). Today, these models have left their place to reference mathematical phantoms in which the complex structure of the human body is better modeled. In the radiation transport calculations performed in this study, the Reference Male Phantom developed by the ICRP was used (Fig. 1). The purpose of using this phantom is to enable the model to be simulated using various MC codes in future studies. $[9,10]$

The ICRP Reference Male Phantom is produced as a result of the creation of the human body using voxels of $2.137 \mathrm{~mm} \times 2.137 \mathrm{~mm} \times 8.0 \mathrm{~mm}$ volume. Each of these small volume voxels are defined using different material contents at atomic scale. Thus, a $176 \mathrm{~cm}$ tall, $73 \mathrm{~kg}$ male human model was defined using 1.9 million voxels. Detailed information about reference phantoms is given in the report numbered 110 of the ICRP.[11]

\section{Dose Calculation Algorithms}

In this study, the algorithms $\mathrm{PB}, \mathrm{CC}$, and $\mathrm{MC}$ are used in the calculation of the radiation distribution.

\section{PB Algorithm}

The $\mathrm{PB}$ algorithm uses a pre-calculated sample photon beam to calculate the dose distribution in water. The algorithm recalculates the dose at different locations using the intensity distribution along the photon moving path. The $\mathrm{PB}$ creates a dose distribution by integrating it at patient's surface to account for changes in primary intensity and changing the shape of the beam by the effect of depth and tissue density of the material. The PB algorithm does not take into account changes in lateral scattering effects.

In other words, PB algorithm is not capable at calculation of the lateral variation of the beam that causes uncertainties.

\section{Algorithm}

The algorithm is capable of the calculating the effects of patient heterogeneities on both primary and secondary scattered radiation. Naturally, it can take into account dose distributions in areas with high electron density variation, such as tissue-air, and tissue-bone.

The algorithm uses an approximation where all energy within a given solid angle will be transported along a line. The choice of the dose calculation algorithm can have a huge impact on a treatment plan for a particular treatment case.[12]

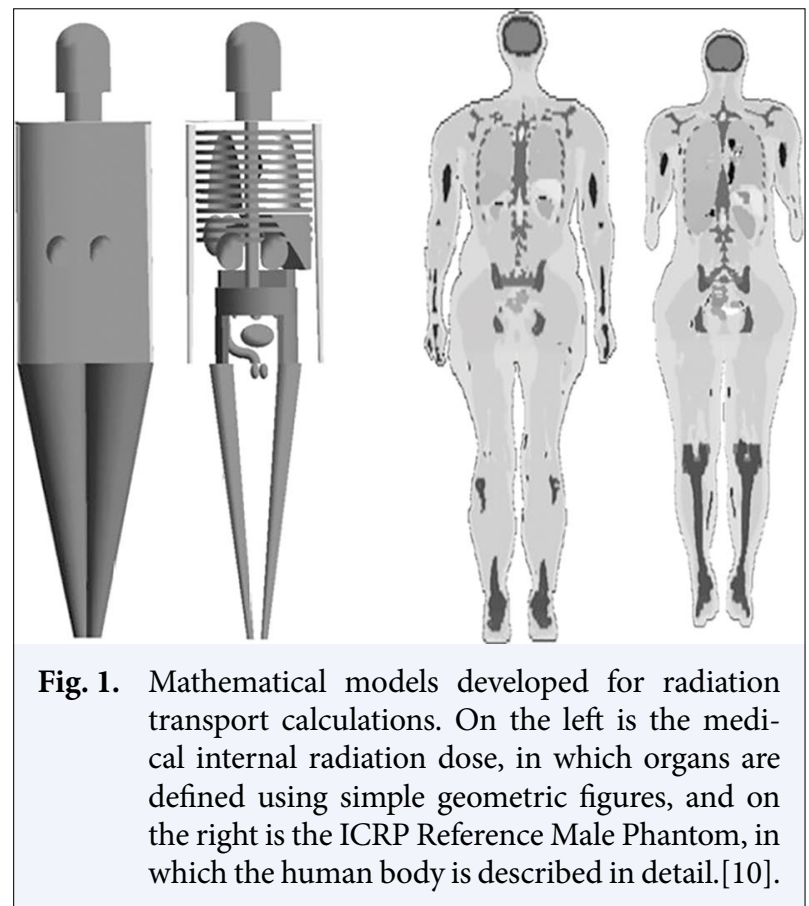




\section{Algorithm}

With the MC method, each photon history (sample) is calculated one by one for a sufficiently high number of primary photons and generated secondaries. Many variance reduction techniques are used to speed up the calculation and decrease the complexity. In this method, a particle history is reused and scaled according to density along its new trajectory. The expected errors correspond to the expansion of the use of applied variance reduction methods. This is used as input data for dose calculations and is itself calculated from a number of air dose profiles.[13]

The Elekta (MONACO/MC) is based on a model using the virtual energy flux model. Dose distribution within the patient is determined by $\mathrm{X}$ ray voxel $\mathrm{MC}$ calculation.[14]

\section{Statistical Analysis}

A two-way analysis of variance (ANOVA) was used to test the differences between different algorithms. All analyses were conducted in SPSS v. 23. Differences were reported to be significant at $\mathrm{p} \leq 0.05$.

\section{Results}

In our study, we observed that different algorithms show different results with the same energy and the same field angles. Table 1 shows statistical evaluations for two-way ANOVA assessing the effect of algorithms and fields with the interaction between the factors. In other words, we assessed the effect on the DVH of two factors (algorithms and fields).

In terms of PTV, the lowest minimum dose (Dmin) and Dmax values were calculated in the CC algorithm. We know that Dmin values are important to assess target volume coverage. Considering that higher Dmin improve the target volume coverage, $\mathrm{MC}$ was the best (high) algorithm because Dmin in PTV was better with the MC algorithm. However, we found very high hot dose values when we evaluated MC algorithm in terms of Dmax. Dmax values occurring at different angles and energies are shown in Figures 2 and 3, respectively. In Figure 2, Dmax values of PTV in 4-field-6 MV energy were 5343 cGy in PB algorithm, 5378 cGy in CC algorithm, and 5548 cGy in MC algorithm. In Figure 3, Dmax values of PTV according to 5- field-18 MV energies were $5215 \mathrm{cGy}$ in PB algorithm, 5172 cGy in CC algorithm, and 5339 cGy in MC algorithm. Dmean values at $6 \mathrm{MV}$ energy were highest in the $\mathrm{MC}$ algorithm and lowest in the $\mathrm{CC}$ algorithm for all angles. $18 \mathrm{MV}$ was the same in
5 fields. However, in 18 MV Dmean values were the highest in PB algorithm and lowest in CC algorithm with 3 , and 4 fields planning.

Considering the $\mathrm{MC}$ and $\mathrm{PB}$ algorithms of the $\mathrm{D} 2$ parameter, which expresses the hot spot formed in our study, we observed that there were significant differences in $6 \mathrm{MV}$ energies. While $\mathrm{D} 2$ values in $6 \mathrm{MV}$ energy were $106.9 \%, 106.1 \%$, and $105.7 \%$ for PB algorithm for 3, 4, and 5 field planning respectively, our data for $\mathrm{MC}$ algorithm were quite high $(111.1 \%, 110.1 \%$, and $108.9 \%$, respectively).We found that the dose difference between the algorithms decreased with the increase in the energy value. In terms of field number, four field doses were lower than 3 and 5 fields $(\mathrm{p}<0.05)$.

In our study, the results in terms of OARs; the max dose value that is important for the spinal cord in the serial organ category was the highest in the MC algorithm and the lowest in the PB algorithm. Considering Dmean values for the heart and lung, which are in the parallel organ category, they were the lowest in the PB algorithm and highest in the MC algorithm. In addition, heart V5 and V 30 values were the lowest in the $\mathrm{PB}$ algorithm, and highest in the MC algorithm. V5, V10, and V20 values for the whole lung were lowest in the $\mathrm{PB}$ algorithm and highest in the MC algorithm.

In terms of $\mathrm{HI}$, no association was found between the algorithms. However, HI values for PB algorithm were the lowest (best homogeneity).

In our study, the most significant difference between the algorithms occurred in the heart (Dmean, V5), lung (Dmean, V5, and V20), PTV (D98), and spinal cord (Dmax) (Table 1).

\section{Discussion}

In the literature review, we did not come across an algorithm study that performed a comprehensive DVH analysis like our study. In this study, we tried to reveal the differences we observed in terms of PTV and OARs in three different algorithms and three different fields/ angles in a heterogeneous region and find if there is any statistical significance. Although we did not aim to measure the accuracy of algorithms, this study reveals how important the statistically significant differences we observed between algorithms would be in clinical practice.

Thoracic region demonstrates differences between algorithms in planning due to the tissues with different densities it contains. Some planning systems require limitations in clinical use because they ignore electron and photon scattering in heterogeneous environments. Especially in tumors such as head and neck and lung 
Table 1 Statistical output for two-way analysis of variance assessing the effect of algorithms and fields with the interaction between the factors

\begin{tabular}{|c|c|c|c|c|c|}
\hline Source & $\begin{array}{l}\text { Type III sum of } \\
\text { squares }\end{array}$ & $\begin{array}{l}\text { Degrees of } \\
\text { freedom }\end{array}$ & Mean squares & F statistic & $\mathbf{p}$ \\
\hline
\end{tabular}

\section{Heart}

Dmean

\section{Algorithms}

Fields

Algorithms*fields V30

Algorithms

Fields

Algorithms*fields

V5

Algorithms

Fields

Algorithms*fields

Lung

Dmean

Algorithms

Fields

Algorithms*fields

V5

Algorithms

Fields

Algorithms*fields V10

Algorithms

Fields

Algorithms*fields V20

Algorithms

Fields

Algorithms*fields

PTV

Dmean

Algorithms

Fields

Algorithms*fields

D98

Algorithms

Fields

Algorithms*fields

D50

Algorithms

Fields

Algorithms*fields

D2

Algorithms

Fields

Algorithms*fields

45556.000
1066260.333
1686.667

56.388
1707.941
47.268

9.427
167.602
1.913

53272.333

191527.000

531.667

59.737

423.474

2.928

27.796

356.356

1.453

12.761

370.866

6.514

30129.778

1491.444

3665.556

73803.111

7694.111

15541.556

24378.111

2771.444

4186.889

77020.111

9340.111

4188.556

22778.000
533130.167
421.667

28.194
853.971
11.817

4.714
83.801
0.478

26636.167

95763.500

132.917

29.869

211.737

0.732

13.898

178.178

0.363

6.380

185.433

1.629

15064.889

745.722

916.389

36901.556

3847.056

3885.389

12189.056

1385.722

1046.722

38510.056

4670.056

1047.139

\subsection{9}

138.310

0.109

1.954

59.182

11.817

91.715

1630.546

9.306

26.243

94.348

0.131

0.000

0.967

34.662

0.000

245.720

0.000

0.849

0.528

13.295

0.002

170.443

0.348

0.000

0.839

6.760

0.016

196.463

0.000

1.725

0.228

3.077

0.096

0.152

0.187

0.861

0.939

11.055

0.004

1.152

0.358

1.164

0.388

2.304

0.156

0.262

0.775

0.198

0.933

4

$\begin{array}{ll}2.193 & 0.168 \\ 0.266 & 0.772 \\ 0.060 & 0.992\end{array}$




\begin{tabular}{|c|c|c|c|c|c|}
\hline Source & $\begin{array}{c}\text { Type III sum of } \\
\text { squares }\end{array}$ & $\begin{array}{l}\text { Degrees of } \\
\text { freedom }\end{array}$ & Mean squares & F statistic & $\mathbf{p}$ \\
\hline \multicolumn{6}{|l|}{ Spinal cord } \\
\hline \multicolumn{6}{|l|}{ Dmax } \\
\hline Algorithms & 312969.000 & 2 & 156484.500 & 61.581 & 0.000 \\
\hline Fields & 25094058.33 & 2 & 12547029.167 & 4937.615 & 0.000 \\
\hline Algorithms*fields & 10912.667 & 4 & 2728.167 & 1.074 & 0.424 \\
\hline \multicolumn{6}{|l|}{$\mathrm{HI}$} \\
\hline Algorithms & 0.001 & 2 & 0.000 & 0.438 & 0.668 \\
\hline Fields & 0.003 & 2 & 0.001 & 1.412 & 0.326 \\
\hline Algorithms*fields & 0.001 & 4 & 0.000 & 0.376 & 0.775 \\
\hline
\end{tabular}

PTV: Planning target volume; $\mathrm{HI}$ : Homogeneity index
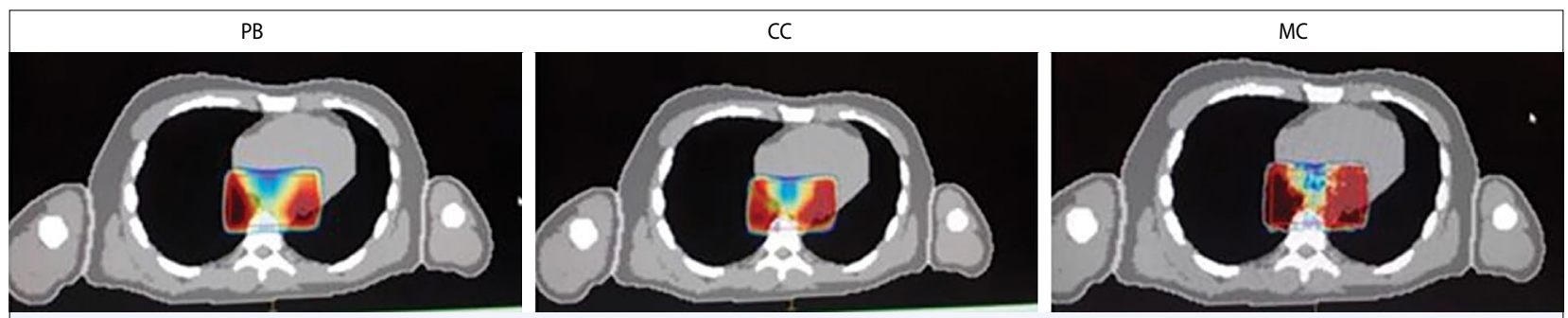

Fig. 2. 4 angles-6MV Dmax
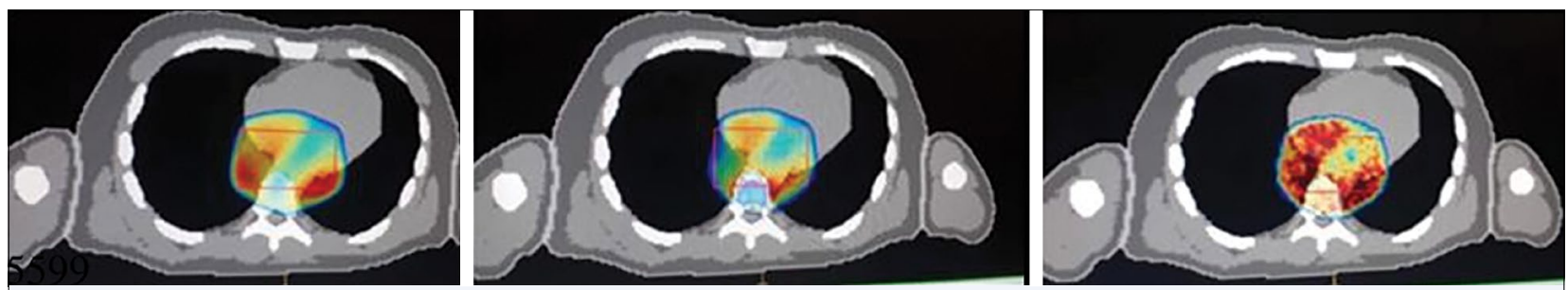

Fig. 3. 5 angels-18MV Dmax.

cancer, dose calculations do not give accurate results. With the technological advances, efforts to improve the dose calculation algorithms used in clinical RT TPSs have increased. Although PB-based algorithms give acceptable results in dose calculations in regions with homogeneous tissue density, some limitations occurred in the utilization of this algorithm, as it does not correctly shape the distribution of secondary electrons formed in heterogeneous tissue density areas. $[15,16]$ In particular, the dose at the lateral interface of tumor and low-density tissue is overestimated by the PB algorithm.[17] Since the $\mathrm{MC}$ algorithm includes all the physical interaction processes that may occur in heterogeneous treatment fields, it is accepted that it performs the most accurate dose calculation in RT.[18,19] It was found suitable for clinical use, such as MC algorithms, in homogeneous and heterogeneous dose calculation studies performed with CC algorithm. [20] In fact, the main issue that worries us as physicians is the increase in the development of loco-regional relapse due to the missing dose at the target and the risk of side effects with high dose values in normal tissues. Pearson et al.[21] compared PB and $\mathrm{CC}$ algorithms and they reported all CC plans had improved dose coverage of the PTV. However, Irvine et al.[22] stated that there are some deficiencies in clinical use in CC algorithm studies. They emphasized that the dose distributions were less homogeneous and that the minimum PTV dose value was up to $23.2 \%$ compared to the PB algorithm. Koelbl et al.[23] compared PB and $\mathrm{CC}$ algorithms in ten lung cancer patients undergoing. The minimum PTV dose value was significantly lower for CC. As a result of measurements, they reported that 
$\mathrm{PB}$ algorithm overestimates the dose at the boundary of the PTV when compared with CC calculation. They emphasized that the $\mathrm{PB}$ algorithm would be critical for RT of lung cancer, since the secondary particles formed were better detected by the CC algorithm. Similarly, in our study, the minimum PTV dose value was up to $10.7 \%$ ( $\mathrm{p}=0.008$ ).However, a meaningful association was not found between PB and MC ( $>>0.05)$.

Kry et al.[24] reported that while PB algorithm overestimated the dose delivered to the center of the target by $4.9 \%$ on average, the $\mathrm{MC}$ algorithm was within $0.6 \%$ on average. They said that this issue should be considered in clinical practice. Since we did not measure the actual dose distributions, we cannot make such an interpretation about the dose differences (deviations from the actual dose) between actual dose and the algorithms. However, based on the data presented in our results, differences between algorithms reveal the importance of dose measurements in terms of target dose and OARs.

In another study, they noted that $\mathrm{PB}$ and $\mathrm{MC}$ algorithms are compatible in terms of target volume, but $\mathrm{MC}$ algorithm is superior to $\mathrm{PB}$ algorithm in a heterogeneous medium or for critical organ dose calculations. [25] The differences between mean doses between the two algorithms were reported to be $0.3 \%$ for PTV, $0.9 \%$ for D50; $2.7 \%$ for D98; and $0.7 \%$ for D2. Furthermore, the differences in terms of bilateral lungs were V5, V10, V20, and MLD were $12.5 \%, 15.8 \%, 14.4 \%$, and $9.1 \%$, respectively. In our data, mean doses for the PTV; the highest difference between PB-MC algorithms at 18MV energy was $1.8 \%$. However, at $6 \mathrm{MV}$ the difference was greater (2.6\%). 1.9\% (18MV energy), $2.6 \%$ (6MV energy) for D50;1.9\% (18MV energy), 1.6\% (6MV energy) for D98; $2.4 \%$ (18MV energy), and 4\% (6MV energy) for D2.

Adigül et al.[26] compared the parameters V5, V13, $\mathrm{V} 20$, and V30 for lung which are especially important in terms of the risk of developing radiation pneumonia in 3DCRT plans they prepared using PB and convolution/ superposition (CS) algorithms. In V5, V13, and V20, higher values were reported in favor of CS and higher values of V30 in favor of PB. Lower PTV Dmin dose values were reported in the CS algorithm. They emphasized that CS algorithm should be preferred due to its higher sensitivity compared to $\mathrm{PB}$, especially in heterogeneous areas such as thorax. Further, in our study, total lung V5 and V20 were higher in CC algorithm compared to PB algorithm (It was higher than the other two algorithms in the MC algorithm). Pearson et al.[21] reported an increase in V20 in the CC algorithm compared to PB. Similarly, according to the data in our study, V20 values are higher in CC than in PB.
As we know, the dose distribution in PTV must be homogeneous and an excess of up to $+7 \%$ isodoses is permitted in routine clinical applications in terms of hot spot (D2). When we examined our study in this respect, we determined that the hot spots formed in the $\mathrm{MC}$ algorithm are at an unacceptable level, especially in plans with 6MV energy.

Koeck et al.[27] evaluated the dose distribution differences between $\mathrm{PB}, \mathrm{CC}, \mathrm{MC}$ algorithms for 3DCRT and IMRT techniques in mediastinal region irradiation. They reported that the PB algorithm overestimated the dose to PTV and high doses to the organs at risk. For 3DCRT, although a well-modeled PB algorithm is clinically acceptable, they suggested using an advanced algorithm such as CC or MC for IMRT planning. In our study, for 3DCRT, when we evaluated in terms of OARs, the lowest values were in $\mathrm{PB}$ and the highest values were in the MC algorithm. PTV mean dose was the highest in PB algorithm at $18 \mathrm{MV}$ energy; it was the highest in $\mathrm{MC}$ algorithm at $6 \mathrm{MV}$ energy.

In summary, in our study, we tried to analyze the effects of different algorithms in the heterogeneous lung tissue, at the angles and energies, we determined. The differences between $\mathrm{PB}$ and $\mathrm{MC}$ algorithms were significantly greater than the differences between CC and $\mathrm{PB}$ algorithms. Based on the data, according to the Quantitative Analyses of Normal Tissue Effects in the Clinic (Ouantec), the algorithm used in dose calculation should also be considered in terms of both PTV and OARs. The subject is important in terms of heterogeneity calculations and clinical applications.

\section{Conclusion}

In this study, we wanted to emphasize that statistically considerable differences can be seen between algorithms used in dose calculation for PTV and OARs. We did not aim to give an answer as to which algorithm is the most correct. The answer to this question will be possible with the increase of clinical algorithm studies evaluated together with the measurement results.

Acknowledgment: We would like to thank the medical physicist Çağrı Yazgan, Orkun Kireç̧̧i, Ali Yeşil and Taylan Tuğrul for their support in planning.

Peer-review: Externally peer-reviewed.

Conflict of Interest: The authors have no conflicts of interest to declare. 
Ethics Committee Approval: This research is a digital phantom study. Patient data were not used in this study (It was done virtually over a computer system).

Financial Support: The authors declared that this study has received no financial support.

Authorship contributions: Concept - T.B.; Design - T.B., B.Ş.Ö.; Supervision - T.B., B.Ş.Ö.; Funding - T.B., B.Ş.Ö.; Materials - T.B.; Data collection and/or processing - T.B.; Data analysis and/or interpretation - T.B., B.Ş.Ö.; Literature search - B.Ş.Ö.; Writing - B.Ş.Ö.; Critical review - B.Ş.Ö.

\section{References}

1. Knöös T, Wieslander E, Cozzi L, Brink C, Fogliata A, Albers D, et al. Comparison of dose calculation algorithms for treatment planning in external photon beam therapy for clinical situations. Phys Med Biol 2006;51(22):5785-807.

2. Arnfield MR, Siantar CH, Siebers J, Garmon P, Cox L, Mohan R. The impact of electron transport on the accuracy of computed dose. Med Phys 2000;27(6):1266-74.

3. Saitoh H, Fujisaki T, Sakai R, Kunieda E. Dose distribution of narrow beam irradiation for small lung tumor. Int J Radiat Oncol Biol Phys 2002;53(5):1380-7.

4. Jones AO, Das IJ. Comparison of inhomogeneity correction algorithms in small photon fields. Med Phys 2005;32(3):766-76.

5. International Commission on Radiation Units and Measurements. Determination of absorbed dose in a patient irradiated by beams of X and Gamma rays in radiation procedures. Bethesda; ICRU Report; 1976.

6. Institution of Physics and Engineering in Medicine and Biology. In: Shaw JE, editor. A guide to commissioning and quality control of treatment planning systems. New York: IPEMB; 1994.

7. Liang $X$, Bradley JA, Zheng D, Rutenberg M, Vega $\mathrm{RM}$, Mendenhall N, et al. The impact of dose algorithms on tumor control probability in intensity-modulated proton therapy for breast cancer. Phys Med 2019;61(5):52-7.

8. Zhuang T, Woody NM, Liu H, Cherian S, Reddy CA, Qi P, et al. Dosimetric differences between local failure and local controlled non-small cell lung cancer patients treated with stereotactic body radiotherapy. A matched-pair study. J Med Imaging Radiat Oncol 2018;62(3):420-4.

9. Goorley T. MCNP ${ }^{\mathrm{mw}}$ 6.1.1-Beta Release Notes (LAUR14-24680). Los Alamos, USA: Los Alamos National Laboratory; 2014.

10. Yeon Y, Han M, Hyeong KC, Hwi J. Conversion of ICRP male reference phantom to polygon-surface phantom. Phys Med Biol 2013;58:6985-7007.
11. Menzel HG, Clement C, DeLuca P. ICRP Publication 110. Realistic reference phantoms: an ICRP/ICRU joint effort. A report of adult reference computational phantoms. Ann ICRP 2009;39(2):1-164.

12. Krieger T, Sauer AO. Monte Carlo-versus pencilbeam-/collapsed-cone-dose calculation in a heterogeneous multi-layer phantom. Phys Med Biol 2005;50(5):859-68.

13. Buzdar SA, Afzal M, Todd-Pokropek A. Comparison of pencil beam and collapsed cone algorithms, in radiotherapy treatment planning for 6 and $10 \mathrm{MV}$ photon. J Ayub Med Coll Abbottabad 2010;22(3):152-4.

14. Bhushan M, Tripathi D, Yadav G, Kumar L, Chowdhary RL, Phuja AK, et al. Feasibility of Monte-Carlo algorithm in comparison with collapse-cone dose calculation algorithm of a commercial treatment planning system in the presence of high-density metallic implant: a dosimetric study. J Egypt Natl Cancer Inst 2021;33(1):2.

15. Hurkmans C, Knöös T, Nilsson P, Svahn-Tapper G, Danielsson H. Limitations of a pencil beam approach to photon dose calculations in the head and neck region. Radiother oncol 1995;37(1):74-80.

16. Engelsman M, Damen E, Koken P, van't Veld AA, van Ingen $\mathrm{KM}$, Mijnheer BJ. Impact of simple tissue inhomogeneity correction algorithms on conformal radiotherapy of lung tumours. Radiother Oncol 2001;60(3):299-309.

17. Knöös T, Ahnesjo A, Nilsson P, Weber L. Limitations of a pencil beam approach to photon dose calculations in lung tissue. Phys Med Biol 1995;40(9):1411-20.

18. Francescon P, Cavedon C, Reccanello S, Cora S. Photon dose calculation of a three-dimensional treatment planning system compared to the Monte Carlo code beam. Med Phys 2000;27(7):1579-87.

19. Vanderstraeten B, Reynaert N, Paelinck L, Madani I, De Wagter C, De Gersem W, et al. Accuracy of patient dose calculation for lung IMRT: a comparison of Monte Carlo, convolution/superposition, and pencil beam computations. Med Phys 2006;33(9):3149-58.

20. Weber L, Nilsson P. verification of dose calculations with a clinical treatment planning system based on a point kernel dose engine. J Appl Clin Med Phys 2002;3(2):73-87.

21.Pearson M, Atherton P, McMenemin R, McDonald F, Mazdai G, Mulvenna P, et al. The implementation of an advanced treatment planning algorithm in the treatment of lung cancer with conventional radiotherapy. Clin Oncol (R Coll Radiol) 2009;21(3):168-74.

22. Irvine $C$, Morgan A, Crellin A, Nisbet A, Beange I. The clinical implications of the collapsed cone planning algorithm. Clin Oncol 2004;16(2):148-54.

23. Koelbl O, Krieger T, Haedinger U, Sauer O, Flentje M. Influence of calculation algorithm on dose distribution 
in irradiation of non-small cell lung cancer (NSCLC) collapsed cone versus pencil beam. Strahlenther Onkol 2004;180(12):783-8.

24. Kry SF, Alvarez P, Molineu A, Amador C, Galvin J, Followill DS. Algorithms used in heterogeneous dose calculations show systematic differences as measured with the Radiological Physics Center's anthropomorphic thorax phantom used for RTOG credentialing. Int J Radiat Oncol Biol Phys 2013;85(1):e95-100.

25. Elcim Y, Dirican B, Yavas O. Dosimetric comparison of pencil beam and Monte Carlo algorithms in conformal lung radiotherapy. J Appl Clin Med Phys 2018;19(5):616-24.

26. Adıgül ŞM, Demiral AN, Karagüler Z. The effect of different calculation algorithms in non-small-cell lung cancer 3-dimensional conformal radiotherapy planning on lung dose-volume parameters. Türk Onkol Derg 2012;27(4):189-201.

27. Koeck J, Abo-Madyan Y, Eich HT, Stieler F, Fleckenstein J, Kriz J, et al. Clinical relevance of different dose calculation strategies for mediastinal IMRT in Hodgkin's disease. Strahlenther Onkol 2012;188(8):653-9. 